\title{
A Portable Optical DSPI System for Residual Stresses Measurement by Hole Drilling Using the Integral Method in Terms of Displacement
}

\author{
Armando Albertazzi G. Jr. ${ }^{1, a^{*}}$, Matias Viotti ${ }^{1, b}$, Celso Veiga ${ }^{1, c}$ \\ ${ }^{1}$ Mechanical Engineering Dept. - Universidade Federal de Santa Catarina, Campus da UFSC, \\ Trindade, Florianópolis, SC, Brazil, CEP 88.050-005, Tel.: +55 (48) 32392030 \\ aa.albertazzi@ufsc.br, bmov@labmetro.ufsc.br, ${ }^{\mathrm{c}} \mathrm{clv} @ l a b m e t r o . u f s c . b r$
}

Keywords: Residual Stress Measurement, Incremental Hole Drilling, ESPI, DSPI, Integral Method

\begin{abstract}
A portable optical device for residual stresses measurement is presented here. It combines an achromatic digital speckle pattern interferometer (DSPI) with radial sensitivity with a high speed pneumatic drilling unit. The system automatically acquires a 1280 x 960 pixel image for each step of incremental hole drilling. The number of drilling steps, as well the drilling increments, is fully programmable. The data is processed and fitted to a set of displacement curves obtained by the Finite Element Method according to the integral formulation. This paper describes the device as well as the data reduction procedure. The system has been applied to measure residual stress profiles in four to eight points of cross-sections of pipes and, from that, to compute the amount of bending moment induced in pipelines used for oil transportation. The paper presents results of a calibration test in a 12 $\mathrm{m}$ long test bench with a $200 \mathrm{~mm}$ steel diameter pipe.
\end{abstract}

\section{Introduction}

Residual stresses measurement using the incremental hole drilling approach with strain gauges is a well-established technique.[1] Despite it is normalized by the ASTM E837 standard [2], the evaluation of a residual stress profile in a single point is a quite time-consuming task. Surface preparation, strain gauge alignment and installation, wiring, drilling alignment, incremental drilling are some required steps that make a complete measurement typically to take an hour or more.

Optical methods are very attractive alternatives for residual stress measurements by the hole drilling method since almost no surface preparation or tedious installations are required. However, most optical systems already available for residual stress measurement are designed to be used inside labs.[3] Optical techniques usually require that the part to be measured must be transported to a laboratory.

The authors have developed a portable optical device for residual stress measurement using the incremental hole drilling approach. [4-6] A highly stable and achromatic digital speckle pattern interferometer (DSPI), capable to measure in radial polar coordinates, is integrated with a high speed drilling unit in a fully computer controlled device for residual stress measurement by incremental hole drilling. A robust construction is designed in order to make it appropriate for in-field measurement. The total measuring time is about 15 to $20 \mathrm{~min}$, which is four times faster than a strain gauge measurement. Similar uncertainty level is achieved. The optical principle, constructive aspects, data reduction approach and one practical application are presented in this paper.

\section{The Radial in-Plane Interferometer}

A $30 \mathrm{~mm}$ diameter binary diffractive optical element (DOE) is the key element of this digital speckle pattern interferometer (DSPI). It consists of a circular binary diffraction grid with a pitch of about $1.30 \mu \mathrm{m}$. Collimated light if diffracted, as it passes through this grid, and bent about $30^{\circ}$, forming a circular double illuminated area of about $10 \mathrm{~mm}$ diameter as it is shown in the left part of Fig. 1.

(c) (1) Content from this work may be used under the terms of the Creative Commons Attribution 3.0 license. Any further distribution of this work must maintain attribution to the author(s) and the title of the work, journal citation and DOI. Published under license by Materials Research Forum LLC. 
The right part of this figure describes the working principle of the DSPI interferometer. The light coming from the laser (L) is expanded by a lens (E). It passes through the elliptical hole of the mirror $\mathrm{M} 1$, reaches mirrors M2 and M3 and is reflected back to the mirror M1. Mirror M1 directs the expanding light to the collimating lens (CL) in order to obtain an annular collimated beam. Finally, the light is diffracted by the DOE mainly in the first diffraction order towards the specimen surface. Mirrors M2 and M3 are two special concentric circular mirrors. The former is joined to a piezoelectric actuator (PZT) and the later has a circular hole with a diameter slightly larger than diameter of M2. Furthermore, mirror M3 is fixed while M2 is mobile. The PZT actuator moves the mirror M2 along its axial direction generating a relative phase difference between the beam reflected by M2 (central beam) and the one reflected by M3 (external annular beam). Every point over the illuminated area receives only one light ray coming from M2 and only other one from M3. Thus, PZT enables the introduction of a relative phase shift for efficient optical phase calculation by means of phase shifting algorithms. The central hole placed at M1 has several functions: (a) to allow that the light coming from the laser source reaches mirrors M2 and M3, (b) to prevent that the laser light reaches directly the specimen surface having triple illumination and (c) to provide a viewing window for the camera (CCD).
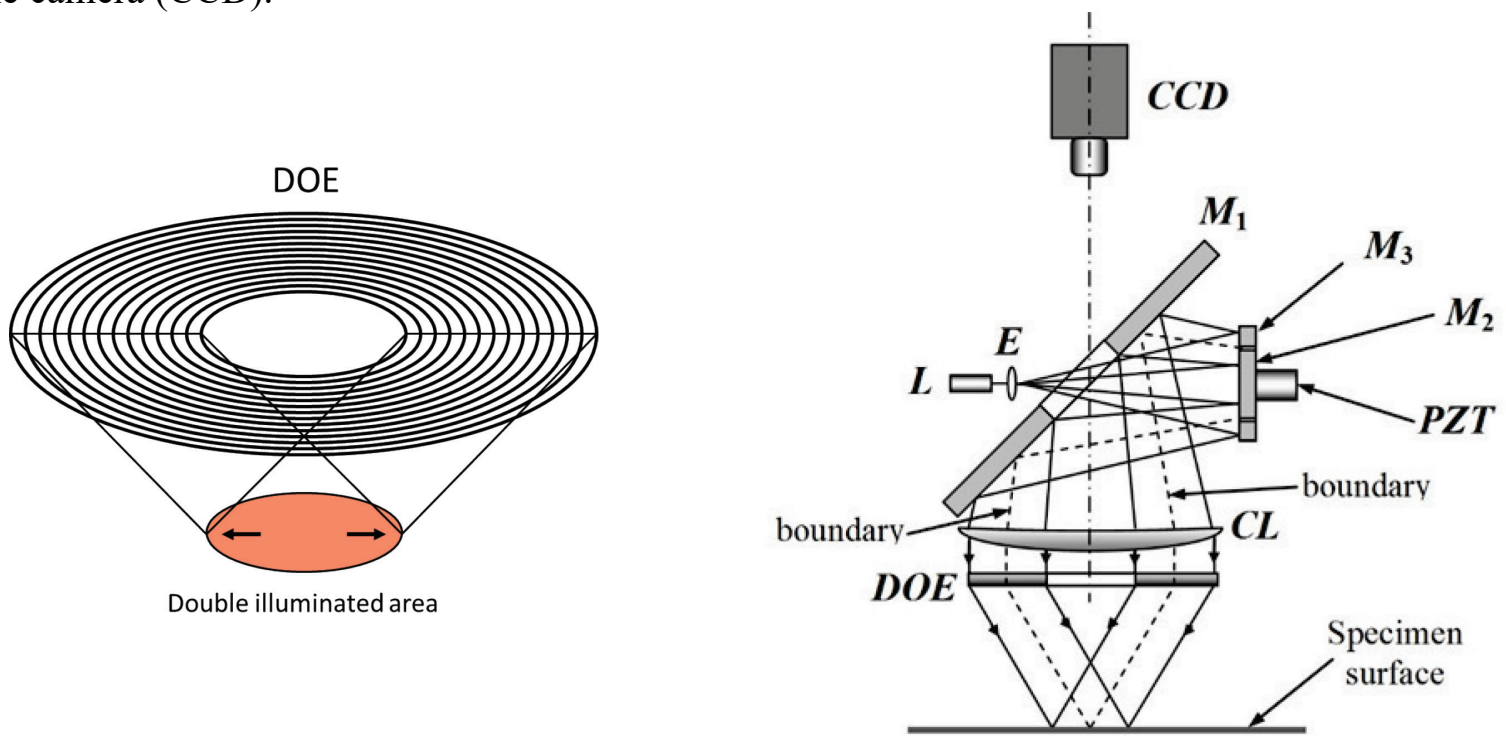

Fig. 1 - Double illumination speckle interferometer with radial in-plane sensitivity.

Since each point on the circular illuminated area is double illuminated, the sensitivity direction is parallel to the surface and aligned with the radial direction. Therefore, only the radial displacement component is measured. This configuration results in an achromatic interferometer, i.e., its sensitivity is not wavelength-dependent. [4] Therefore, cheap and compact diode lasers can be used as light source without stability loss.

\section{The Integrated Device}

Fig. 2 shows in-field applications of the radial in-plane DSPI interferometer integrated with a pneumatic high-speed drilling unit in a portable residual stresses measurement device. [4-6] The outof-lab application is possible since the relative motions between the device and the part to be measured are minimized by a highly stiff clamping. Three legs, with sharp conical tips of hardened steel, are provided for leveling and stablishing a well-defined and firm binding interface to the specimen surface. A set of four rare earth magnets develops the attraction forces to firmly press the three legs against the specimen surface. A motorized rotating stage is used for automatically exchanging the measuring unit and the drilling unit. An integrated measurement software automatically controls all steps of an incremental hole-drilling measurement and processes all necessary images to compute the combined stresses profile as a function of the surface depth. 

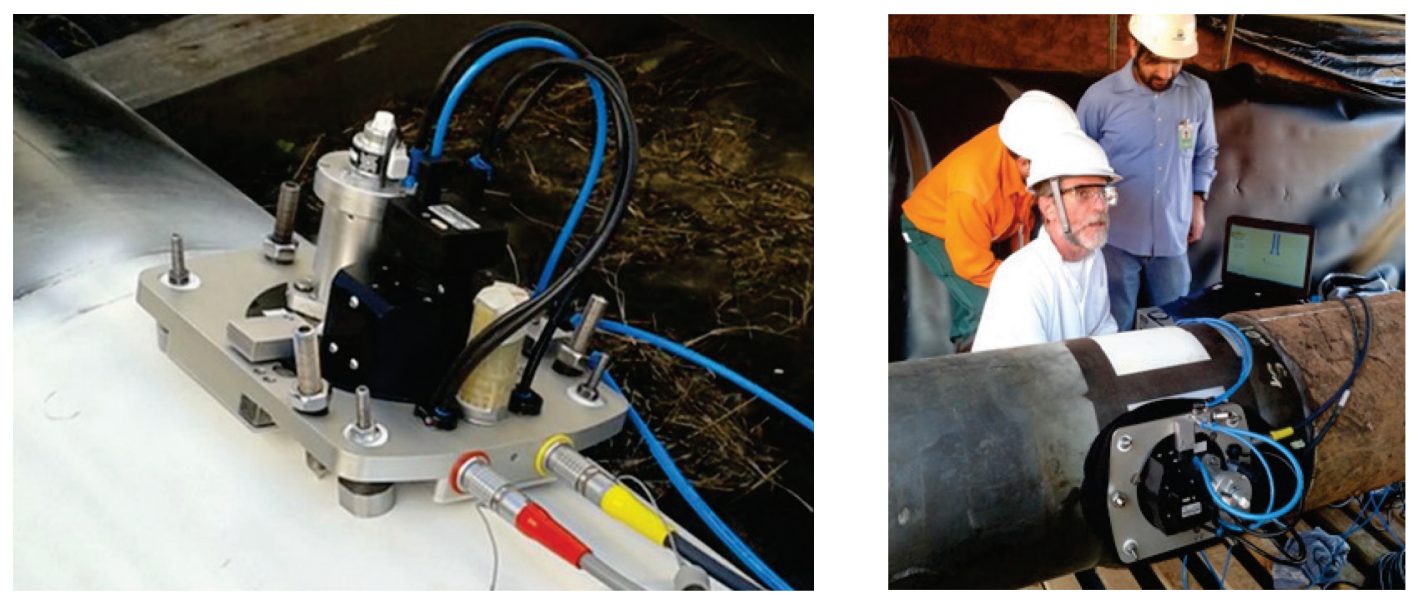

Fig 2. In-field applications of the integrated DSPI/hole drilling device.

The surface to be measured needs to be clean, without any thick coating and reasonably smooth. If the surface is very dark, or shining, a very thin layer of light spray painting is recommended.

\section{Image Processing and Data Reduction}

The left part of the Fig. 3 shows a typical DSPI phase difference pattern. It was obtained after drilling a hole with $1.6 \mathrm{~mm}$ in diameter and $1.0 \mathrm{~mm}$ in depth into a specimen with uniaxial residual stresses along the horizontal axis. The corresponding radial displacement signal was extracted along the constant radius line (light blue) drawn in the left part this figure. The elliptical line corresponds to the measured signal expressed in polar coordinates in a much-enlarged scale. The right part of the figure shows the same radial signal now expressed in Cartesian coordinates. The horizontal axis corresponds to the angle in degrees and the vertical axis is the radial displacement component. Note that, due to image symmetry, the radial displacement signal is sinusoidal with two periods along $360^{\circ}$.
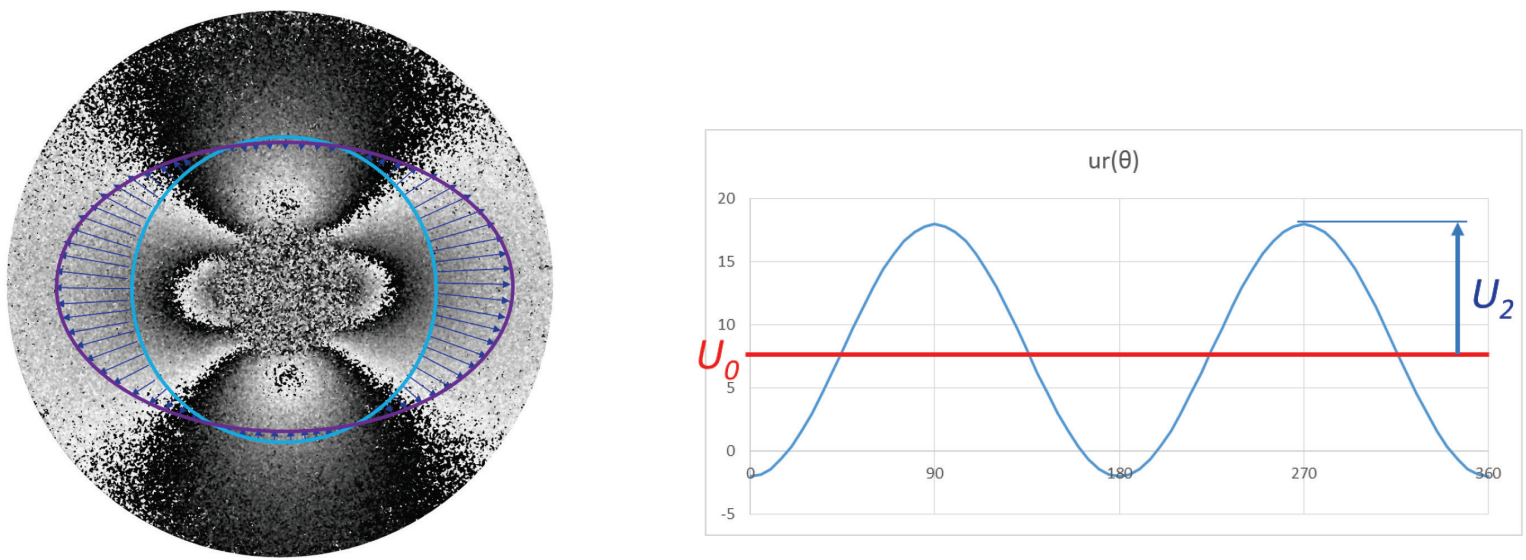

Fig 3 - Left: typical hole drilling image. Right: Signal extracted along a constant radius line.

The general equation for the radial displacement component $\left(u_{r}\right)$ is like Eq. 1. For a given radius, this equation corresponds to the right part of Fig. 3. There are two signal components: $U_{0}$, the mean value and a sinusoidal component with two waves and amplitude $U_{2}$. The signal component $U_{0}$ is further referred as the zero harmonic and is related to the residual stresses sum $\sigma_{1}+\sigma_{2}$. The signal $U_{2}$, is the second harmonic and is related to the residual stresses difference $\sigma_{1}-\sigma_{2}$. Both quantities vary for different values of the radius $r$. Therefore, they are functions of $r$. The angle $\beta$ corresponds to the principal stress direction.

$$
u_{r}(r, \theta)=U_{0}(r)+U_{2}(r) \cos (2 \theta-2 \beta)
$$


Eq. 2 has a more convenient relationship, where $U_{0}$ is replaced by $a \cdot\left(\sigma_{1}+\sigma_{2}\right)$ and $U_{2}$ by $b \cdot\left(\sigma_{1}-\sigma_{2}\right)$. Both $a(r)$ and $b(r)$ are the normalized radial displacement responses for the unitary stress sum and difference, respectively. They depend on the hole diameter, the hole depth and the material properties.

$$
u_{r}(r, \theta)=a(r)\left(\sigma_{1}+\sigma_{2}\right)+b(r)\left(\sigma_{1}-\sigma_{2}\right) \cos (2 \theta-2 \beta)
$$

\section{Residual Stresses Calculation}

The functions $a(r)$ and $b(r)$ can be determined numerically to a set of discrete radius values $\left(r_{k}\right)$. To quantify the amount of residual stresses from the measured quantities $U_{0}\left(r_{k}\right)$ and $U_{2}\left(r_{k}\right)$ a linear behavior is assumed. Therefore, the stress values are proportional to the amount of radial displacement. As an example, let's suppose that $U_{0}\left(r_{k}\right)$ is $P$ times bigger than $a\left(r_{k}\right)$. In this case, the sum of residual stresses will be $P$ times bigger than $1.0 \mathrm{MPa}$. Since random errors are expected in the experimental values $U_{0}\left(r_{k}\right)$ as well as an unexpected bias $C$, the relationship between $U_{0}\left(r_{k}\right)$ and $a\left(r_{k}\right)$ is in the form of Eq.3, where $\varepsilon\left(r_{k}\right)$ is a random error component. The $k$ sub index was omitted here for simplicity. The values of $P$ and $C$ can be determined by a least square fitting. [7] A similar approach is used to relate $U_{2}\left(r_{k}\right)$ and $b\left(r_{k}\right)$, but it will not be described here.

$$
U_{0}(r)=P \cdot a(r)+C+\varepsilon(r)
$$

Indeed, a set of discrete numerical normalized signals are required to apply the integral method for the residual stresses determination by the incremental hole drilling. Each numerical signal is related to the hole depth $(i)$ and the stress layer $(\mathrm{j})$. Let $a_{i j}\left(r_{k}\right)$ denotes the numerical signal computed for the $i$-depth and $j$-stress layer [8]. For the first hole depth, the relationship between the experimental data $U_{0}\left(r_{k}\right)$ and numerical signal $a_{11}\left(r_{k}\right)$ is given by Eq. 4 . Also here, the $k$ sub index was omitted.

$$
U_{01}(r)=P_{1} \cdot a_{11}(r)+C_{1}+\varepsilon_{1}(r)
$$

For the second incremental hole depth, the integral method expects to relate the experimental signal to the numerical one according to Eq. 5. Since Eq. 4 alone can determine the value of $P_{1}$, Eq. 5 involves two additional unknowns: $P_{2}$ the stress value for depth 2 and the additive constant $C_{2}$.

$$
U_{02}(r)=P_{1} \cdot a_{21}(r)+P_{2} \cdot a_{22}(r)+C_{2}+\varepsilon_{2}(r)
$$

It is straightforward extending Eq. 5 for an integer number of $S$ hole increments. The least squares solution for the $P_{i}$ values can be derived and results in a $S$ x $S$ system with the $S$ unknowns: $P_{1}$ to $P_{S}$. The resulting matrix system can be written as Eq. 6 where the elements of the matrix $[X]$ and vectors $\{P\}$ and $\{Y\}$ are given in Eq. 7 .

$$
\begin{aligned}
& {[X]\{P\}=\{Y\}} \\
& \{P\}=\left\{\begin{array}{c}
P_{1} \\
\vdots \\
P_{S}
\end{array}\right\} \quad X_{m, n}=\sum_{i=Q}^{S}\left[\sum_{\text {all } r}\left(a_{\text {im }} \cdot a_{\text {in }}\right)-\frac{1}{N} \sum_{\text {all } r} a_{\text {im }} \cdot \sum_{\text {all } r} a_{\text {in }}\right]
\end{aligned}
$$




$$
Y_{m}=\sum_{i=Q}^{S}\left[\sum_{\text {all } r}\left(a_{i m} \cdot U_{0 i}\right)-\frac{1}{N} \sum_{\text {all } r} a_{\text {im }} \cdot \sum_{\text {all } r} U_{0 i}\right]
$$

The integer numbers $m$ and $n$ are the row and column indexes of each matrix element. The quantity $Q$ is defined as the minimum integer between $m$ or $n: Q=\min (m, n) . N$ is the number of discrete radial values related to the index $k$ (omitted in Eq. 6 and 7).

\section{Bending Moment Calculation}

The combined hole drilling DSPI system was used to determine the amount of bending stress developed in a $12 \mathrm{~m}$ long and $200 \mathrm{~mm}$ (8") diameter steel pipe [7, 9]. Left part of Fig. 4 shows a sketch of the test bench with two parallel pipes. They were clamped at both ends and a hydraulic jack was used to apply a transverse force measured by a load cell as $(37621 \pm 58) \mathrm{N}$ at the central point. Bending moments were measured by the hole drilling method in eight different cross-sections of the right pipe in Fig. 4 and compared with the reference values computed from the central load value. The sample pipe is the right one in the figure.
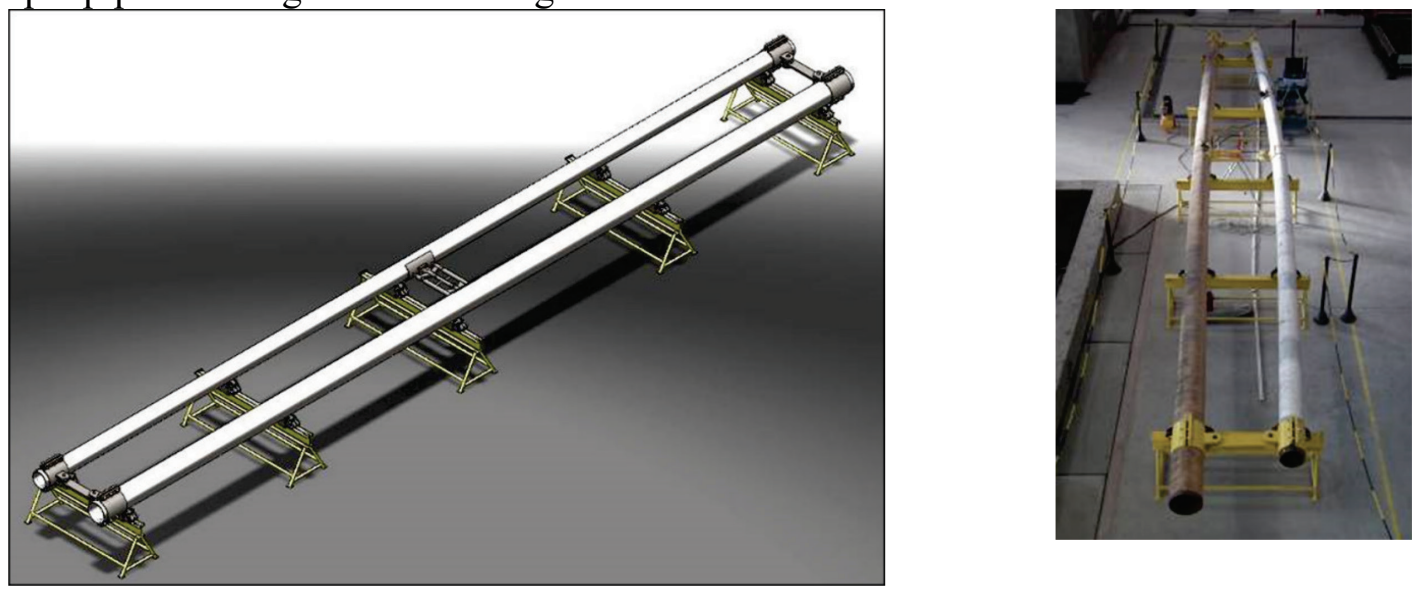

Fig 4-12 m long steel pipe loaded by a known bending moment.

To determine the bending moments, a set of eight hole drilling measurements was carried out in each cross-section at different angles separated by $45^{\circ}$. The holes were drilled in 20 steps, from 0.00 to $1.00 \mathrm{~mm}$ with depth increments of $0.05 \mathrm{~mm}$. The phase images obtained in each final drilling step are shown in Fig. 5.

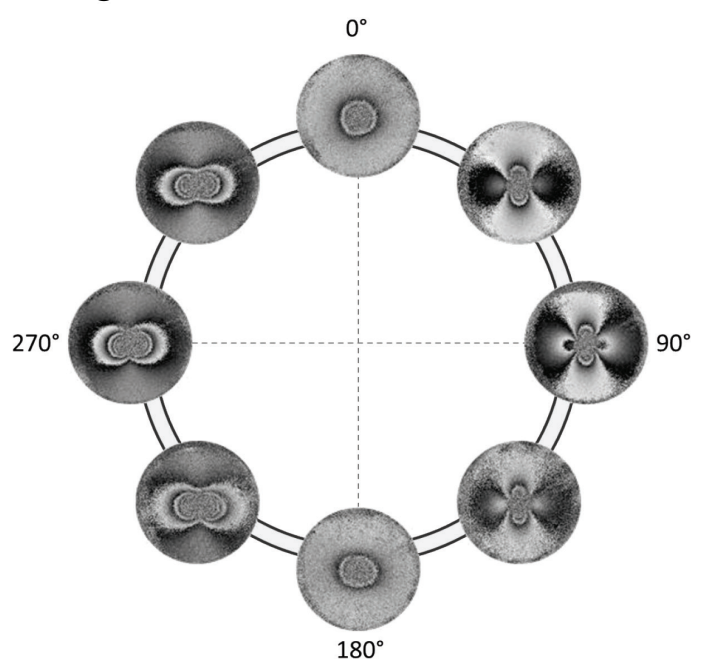

Fig 5 - Different residual stress measurements at eight different angles of a same pipe crosssection. 
A compressive residual stresses peak value of $-200 \mathrm{MPa}$ was typically obtained at the depth of $0.25 \mathrm{~mm}$ due to the pipe manufacturing process. The different residual stresses profile offsets in the pipe axis direction were used to determine the amount of bending stresses. The bending stress in each cross-section varied from $70 \mathrm{MPa}$ to $340 \mathrm{MPa}$, depending on the angular location of the measurement. The agreement between the central values determined by the hole drilling method and the reference ones were in the range of $\pm 20 \mathrm{MPa}$.

\section{Conclusions}

The authors developed a portable optical system with a radial in-plane achromatic interferometer for residual stresses measurement by the incremental hole drilling technique using the integral method. The total setup and measurement time ranged from 15 to $20 \mathrm{~min}$ for a complete 20 steps incremental hole drilling measurement. No special skills are required from the user. The paper presented results from bending stresses measurement in a $12 \mathrm{~m}$ long and $200 \mathrm{~mm}$ diameter pipe subjected to a known amount of bending moment. The central values for the bending stresses obtained for eight different cross-sections were compared with the reference values computed from the load cell and bending bench geometry. The deviations were all inside the range of $\pm 20 \mathrm{MPa}$.

\section{References}

[1] Schajer, G. S. (Editor), "Practical Residual Stress Measurement Methods", Wiley 2013, 7, ISBN 9781118342374.

[2] ASTM E837-13. "Standard test method for determining residual stresses by the hole-drilling strain-gage method", Annual Book of ASTM Standards. American Society for Testing and Materials (2013).

[3] Schajer, G.S. and Rickert, T.J., "Incremental Computation Technique for Residual Stress Calculations Using the Integral Method", Exp. Mech., 51(7), 1217-1222 (2011). http://dx.doi.org/10.1007/s11340-010-9408-5

[4] Viotti, M. R., Kapp W. and Albertazzi Jr., A. "Achromatic digital speckle pattern interferometer with constant radial in-plane sensitivity by using a diffractive optical element," App. Opt. 48(12), 2275-2281 (2009). http://dx.doi.org/10.1364/AO.48.002275

[5] Viotti, M. R., Albertazzi Jr., A. and Kapp, W. A., "Experimental comparison between a portable DSPI device with diffractive optical element and a hole drilling strain gage combined system," Opt. Lasers Eng. 46 (11), 835-841 (2008). http://dx.doi.org/10.1016/j.optlaseng.2008.05.020

[6] Viotti, M. R. and Albertazzi Jr., A. "Compact sensor combining digital speckle pattern interferometry and the hole-drilling technique to measure non-uniform residual stress fields," Opt. Eng. 52 (10), 101905.1-101905.8 (2013).

[7] Albertazzi Jr, A.; Zanini, F. ; Viotti, M. R. ; Veiga, C. L. N. "Residual stresses measurement by the hole-drilling technique and DSPI using the integral method with displacement coefficients". In: $5^{\text {th }}$ International Symposium on Experimental Mechanics e 9th Symposium on Optics in Industry, 2015, v. 1. p. 1-7.

[8] Schajer, G. S., "Measurement of Non-Uniform Residual Stresses Using the Hole-Drilling Method. Part I - Stress Calculation Procedures", J. Eng. Mater. Technol.. 1988; 110(4):338-343. doi: 10.1115/1.3226059. http://dx.doi.org/10.1115/1.3226059

[9] Pacheco, A.; Fontana, F.; Viotti, M. R. ; Veiga, C. L. N. ; Lothhammer, L. R. ; Albertazzi Jr, A. "Bending moment evaluation of a long specimen using a radial speckle pattern interferometer in combination with relaxation methods". In: SPECKLE 2015: International Conference on Speckle Metrology, 2015, Guanajuato. SPECKLE 2015. Guanajuato: CIO, 2015. v. 1. 